\title{
Hepatopatía crónica, edemas y ascitis, ¿puede esconder algo más?
}

\author{
Gema Navarro-Jiménez' ${ }^{1}$ Elena Batlle-López² \\ 'Servicio de Medicina Interna. Hospital Universitario Fundación Alcorcón. Alcorcón (Madrid). España \\ ${ }^{2}$ Servicio de Cardiología. Hospital Universitario Fundación Alcorcón. Alcorcón (Madrid). España
}

Recibido: 29/10/2018

Aceptado: 03/12/2018

En línea: 31/12/2018

Citar como: Navarro-Jiménez G, Batlle-López E. Hepatopatía crónica, edemas y ascitis, ipuede esconder algo más? Rev Esp Casos Clin Med Intern (RECCMI). 2018 (Dic); 3(3): 130-132. doi: 10.32818/reccmi.a3n3a10.

Autor para correspondencia: Gema Navarro-Jiménez.gema.nvj@gmail.com

\section{Palabras clave \\ $\triangleright$ Anamnesis \\ $\triangleright$ Miocardiopatía hipertrófica \\ $\triangleright$ Alcoholismo \\ $\triangleright$ Ascitis}

\begin{abstract}
Resumen
Presentamos el caso de un varón de 47 años con diagnóstico previo de hepatopatía crónica enólica que ingresa por primer episodio de descompensación edemo-ascítica. Tras varios datos en contra del origen de la hepatopatía como causa de la clínica, se llegó al diagnóstico de miocardiopatía hipertrófica familiar en fase de disfunción ventricular. Es un ejemplo de la importancia de integrar una valoración clínica adecuada con las pruebas complementarias que en ocasiones pueden ser confusas y retrasar el diagnóstico final.

Abstract
We present the case of a 47-year-old man with a previous diagnosis of chronic enolic liver disease who was admit-
ted for the first episode of edemo-ascitic decompensation. After several data against the origin of liver disease as a
cause of the clinical manifestations, diagnosis of familial hypertrophic cardiomyopathy in the phase of ventricular
dysfunction was reached. It is an example of the importance of integrating an adequate clinical assessment with
complementary tests that can sometimes be confusing and delay the final diagnosis.
\end{abstract}

Acudió a Urgencias por edemas en piernas y aumento progresivo del perímetro abdominal con astenia y plenitud pospandrial. No refería otros síntomas. A la exploración física, estaba normotenso y taquicárdico, afebril. Aceptable estado general, bien perfundido, eupneico y tolerando decúbito. La presión venosa yugular estaba elevada hasta ángulo mandibular a $90^{\circ}$. La auscultación cardíaca era rítmica con soplo sistólico suave en borde esternal derecho, la auscultación pulmonar normal. Presentaba abdomen distendido mate en flancos con oleada ascítica, hepatomegalia ligeramente dolorosa y latido hepático sin soplo. En miembros inferiores presentaba edemas hasta raíz de muslos, pulsos distales presentes.

\section{Evolución y pruebas complementarias}

Se trata de un varón de 47 años que ingresa por primer episodio de descompensación hidrópica. Entre sus antecedentes personales destacaba: ex fumador de 20 paquetes/año y consumidor hasta 2014 de $60 \mathrm{~g}$ de alcohol/día, hiperuricemia y seguimiento en Digestivo por hepatopatía crónica de probable origen enólico A6 de Child. Diagnosticado de fibrilación auricular (FA) en 2007 con ecocardiograma transtorácico (ETT) con hipertrofia ventricular izquierda, función sistólica ventricular izquierda (FEVI) conservada. Como antecedentes familiares refería padre diagnosticado en la juventud de FA y cardiopatía no filiada y, finalmente, fallecimiento por causa cardiológica.

Estaba en tratamiento con alopurinol, digoxina, bisoprolol y acenocumarol.
En Urgencias se realizaron analítica con hemograma, bioquímica con perfil hepático básico y función renal normales. En la radiografía de tórax tenía un índice cardiotorácico aumentado, sin otros hallazgos. Se describía un electrocardiograma (ECG) con FA a 105 latidos por minuto (lpm). Se realizó paracentesis diagnóstica con líquido peritoneal compatible con trasudado y gradiente

Ingresó en Medicina Interna con diagnóstico de primer episodio de descompensación hidrópica en paciente con hepatopatía crónica probablemente seroascítico de albúmina de 0,9. 
enólica. Al ingreso, algunos datos hacían dudar de que la descompensación fuera secundaria a hepatopatía: la marcada elevación de la presión venosa yugular y un gradiente seroascítico de albúmina $<1,1$ hacían poco probable que la ascitis fuera por hipertensión portal ${ }^{1}{ }^{2}$. Se solicitó analítica con NT-proBNP de $1.800 \mathrm{ng} / \mathrm{l}, \mathrm{GGT}$ de $94 \mathrm{U} / \mathrm{l}$ y fosfatasa alcalina levemente elevada al igual que la bilirrubina. Con estos hallazgos, se decidió solicitar estudio cardiológico con nuevo ECG y ETT. El ECG (Figura 1) mostraba FA a 70 lpm eje derecho, bloqueo de rama derecha, Q septal, en I y AVL y bajo voltaje en derivaciones de los miembros. En el ETT presentaba ventrículo izquierdo (VI) no dilatado con hipertrofia severa septal $(15 \mathrm{~mm})$, moderada del resto (13-14 mm). Sin gradiente dinámico en tracto de salida del VI, ni basal ni tras Valsalva. Miocardio sin aspecto que sugiera afectación específica. FEVI límite, sin alteraciones segmentarias. Dilatación aneurismática de ambas aurículas. Presiones izquierdas elevadas. Insuficiencia mitral moderada. Ventrículo derecho moderadamente dilatado con función contráctil levemente deprimida. Insuficiencia tricúspide severa. Cava inferior dilatada $(31 \mathrm{~mm})$ sin colapso inspiratorio. Hipertensión pulmonar severa (65 mmHg). Derrame pericárdico ligero sin repercusión. Test de burbujas negativo.

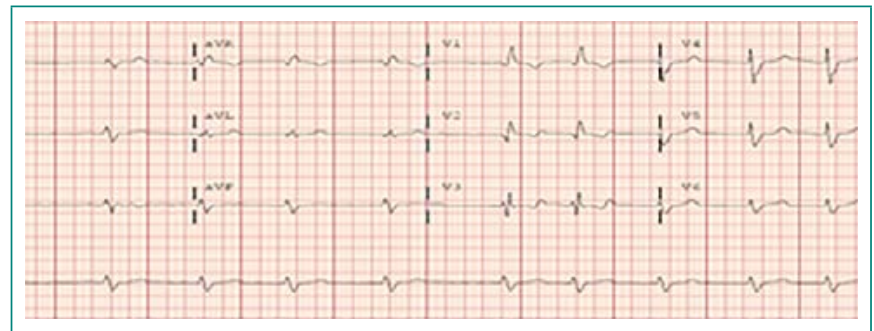

Figura 1. ECG con Q septal y en AVL y bajo voltaje en derivaciones de los miembros

Dados los antecedentes familiares del paciente, la presencia de hipertrofia ventricular izquierda en paciente no hipertenso junto con ECG sugerente de miocardiopatía infiltrativa (ondas Q de pseudonecrosis y bajo voltaje en derivaciones de los miembros), se amplió el estudio con la realización de proteinograma en sangre y orina que fueron normales y resonancia magnética (RM) cardíaca (Figura 2). La RM fue informada como patrón de realce en septo interventricular medio y en los puntos de anclaje con el ventrículo derecho (VD) sugestivo de hipertensión pulmonar versus miocardiopatía hipertrófica en fase avanzada de disfunción ventricular (FEVI 44\%). Signos de hipertensión pulmonar. Se decidió finalmente solicitar test genético de miocardiopatías restrictivas, identificándose una variante patogénica en MYPBC3 (c.2149-1G>A) para el desarrollo de miocardiopatía hipertrófica.

Tras tratamiento con diurético intravenoso a dosis altas y en perfusión continua, hubo un ligero deterioro de la función renal, por lo que se añadió perfusión de levosimendán con excelente respuesta clínica, lográndose un balance negativo durante el ingreso de unos 20 litros con resolución de los datos de insuficiencia cardíaca. Toleró bien el paso a diurético vía oral y fue dado de alta añadiendo a su tratamiento torasemida y eplerenona. Se remitió a consulta de trasplante cardíaco.

\section{Diagnóstico}

Miocardiopatía hipertrófica en fase de disfunción ventricular. Hipertensión pulmonar severa. IT severa. Insuficiencia cardíaca severa de predominio derecho con hígado de estasis.

\section{Discusión}

Hemos querido presentar este caso porque aporta aspectos interesantes para reflexionar. Por un lado, la importancia de la valoración integral en la orientación diagnóstica de una patología concreta. Al tratarse de un paciente ex enólico se interpretó la hepatopatía como secundaria a este hábito. Sin embargo, tras revisar exploraciones, observamos que, ya desde 2012, en ecografías abdominales describían cava inferior y suprahepáticas dilatadas; además, nunca se habían descrito datos de hipertensión portal. Se podría haber realizado un despistaje más precoz de la cardiopatía para evitar la sobrecarga crónica de volumen con daño hepático establecido. También, se plantea el diagnóstico diferencial de cardiopatía hipertrófica familiar. Según la guía de miocardiopatía hipertrófica de 2014 de la European Society of Cardiology (ESC)³, la clasificación está basada en criterios morfológicos, y agrupan las etiologías en familiares/con base genética y no familiares/sin base genética. La mayoría de veces tiene base genética, agrupándose en mutaciones en genes de proteínas sarcoméricas (40-60\%), donde MYBPC3 y MYH7 son las más frecuentes; mutaciones en otros genes no sarcoméricos (5-10\%), donde se incluirían trastornos metabólicos (enfermedad de Fabry, enfermedad de Danon...), enfermedades neuromusculares, amiloidosis familiar relacionada con transtirretina y síndromes malformativos (Noonan, LEOPARD...) y, por último, causas sin base genética como algunas amiloidosis y trastornos endocrinos

En nuestro paciente, su padre era el probable familiar afecto, quedando razonablemente descartadas las enfermedades ligadas al cromosoma X. Inicialmente, se enfocó el diagnóstico hacia miocardiopatía infiltrativa por el ECG con bajo voltaje en derivaciones de miembros y Q de pseudonecrosis, hallazgos discordantes con la presencia de hipertrofia ventricular izquierda ecocardiográfica. Sin embargo, las ondas Q patológicas se observan hasta en el 20-30\% de pacientes con miocardiopatía hipertrófica, sobre todo en derivaciones inferiores y laterales, aunque suele ir acompañado de voltajes altos ${ }^{4}$. El bajo voltaje electrocardiográfico tras descartar hipotiroidismo, obesidad, derrame pericárdico significativo y enfisema pulmonar es sugestivo de miocardiopatía infiltrativa, aunque no patognomónico. Se observa también en pacientes con miocardiopatía hipertrófica en fase avanzada probablemente por la presencia de fibrosis miocárdica.
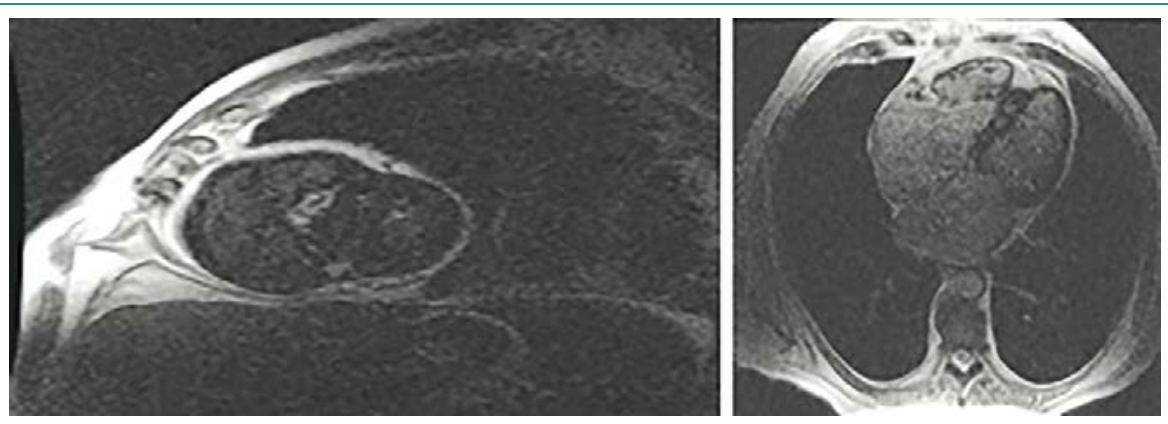

Figura 2. RM cardíaca: patrón de realce en septo interventricular medio y en los puntos de anclaje con VD
Aunque el patrón oro en el diagnóstico diferencia de las miocardiopatías es la biopsia endomiocárdica ${ }^{3}$ la RM permite realizar el diagnóstico diferencial entre la hipertrofia ventricular izquierda no específica como la hipertensiva frente a la miocardiopatía hipertrófica o las miocardiopatías infiltrativas con patrones característicos ${ }^{5}$.

El diagnóstico de certeza se estableció mediante estudio genético que mostró una mutación característica de la miocardiopatía hipertrófica y ausencia de mutaciones de miocardiopatías infiltrativas. 
En conclusión, la valoración adecuada de un paciente requiere integrar una buena historia clínica y exploración física con las exploraciones complementarias; esto evita retrasos en el diagnóstico y permite instaurar el tratamiento más adecuado para la patología de base.

\section{Bibliografía}

1. Thomsen T, Shaffer R, White B, Setnik GS. Paracentesis. N Eng J Med. 2006; 355: e21.

2. European Association for the Study of the Liver. EASL clinical practical guidelines on the management of ascites, spontaneous bacterial peritonitis, and hepatorenal syndrome in cirrhosis. J Hepatology. $2010 \mathrm{Sep}$; 53(3): 397-417. doi: 10.1016/j.jhep.2010.05.004.
3. Elliott PM, Anastasakis A, Borger MA, Borggrefe M, Cecchi F, Charron P, et al. 2014 ESC Guidelines on diagnosis and management of hypertrophic cardiomyopathy. Eur Heart J. 2014 Oct 14; 35(39): 2733-2779. doi: 10.1093/ eurheartj/ehu284.

4. Pérez Riera AR, Barbosa Barros R. Hypertrophic cardiomyopathy: value of electrocardiogram for the diagnosis of different types and for differential diagnosis with athlete's heart. Rev Fed Arg Cardiol. 2015; 44(1) 12-24.

5. Dumont $C A$, Monserrat $L$, Soler R, Rodríguez E, Fernández X, Peteiro J, Bouzas B, Piñón P, Castro-Beiras A. Significado clínico del realce tardío de gadolinio con resonancia magnética en pacientes con miocardiopatía hipertrófica. Rev Esp Cardiol. 2007; 60(1): 15-23. Doi: 10.1157/13097921. 